\title{
Label Preference Schemes for Lightpath Provisioning and Restoration in Distributed GMPLS Networks
}

\author{
Alessio Giorgetti, Nicola Sambo, Student Member, IEEE, Isabella Cerutti, Member, IEEE, Nicola Andriolli, and \\ Piero Castoldi, Member, IEEE
}

\begin{abstract}
In wavelength-routed networks based on a GMPLS control plane, the resource reservation protocol with traffic engineering extensions (RSVP-TE) allows to establish end-to-end lightpaths. The resource reservation can be blocked due to lack of available resources (forward blocking) or due to resource contentions (backward blocking). In wavelength-routed networks, the backward blocking is the predominant blocking contribution, when traffic load is low or highly-dynamic and when lightpath restoration takes place. To reduce the backward blocking, the paper proposes two label preference (LP) schemes compliant with RSVP-TE message exchanges. LP schemes provide the destination node with a label identifying the preferred wavelength to reserve. The preferred label is computed in a distributed way during the forward signaling phase, with the objective of assigning disjoint wavelengths to reservation attempts that may contend the resources. Simulation results demonstrate that, compared to other schemes, LP schemes are effective in reducing the backward blocking during both lightpath provisioning and restoration, without negatively impacting the forward blocking.
\end{abstract}

Index Terms-Backward blocking, dynamic restoration, forward blocking, GMPLS control plane, provisioning, RSVP-TE, wavelength-routed networks.

\section{INTRODUCTION}

$\mathbf{I}$ N WAVELENGTH-ROUTED NETWORKS, where transparent optical nodes (e.g., optical cross-connects) are interconnected by wavelength-division multiplexed (WDM) links, high capacity connections (i.e., lightpaths) can be established on-demand directly in the optical domain. To dynamically accommodate a lightpath request, a route and a wavelength on each link are opportunely selected, in real time, by the network control plane. Since it is difficult to rapidly refresh the information at each node of the network, a control plane with distributed (i.e., local) knowledge of the resource availability is strongly recommended.

The protocol suite currently used in distributed control planes for wavelength-routed networks is the generalized multiprotocol label switching (GMPLS) [1], where the physical wavelengths on each network link are represented by generalized labels. Within GMPLS protocol suite, lightpaths are

Manuscript received March 9, 2007; revised August 8, 2007. Current version published April 17, 2009. This work was supported by the BONE-Project ("Building the Future Optical Network in Europe"), a Network of Excellence funded by the European Commission through the 7th ICT-Framework Programme.

The authors are with Scuola Superiore Sant'Anna, Pisa, Italy (e-mail: alessio.giorgetti@sssup.it; nicola.sambo@sssup.it; isabella.cerutti@sssup.it; nicola.andriolli@sssup.it; piero.castoldi@sssup.it).

Color versions of one or more of the figures in this paper are available online at http://ieeexplore.iee.org.

Digital Object Identifier 10.1109/JLT.2008.917380 established using the resource reservation protocol with traffic engineering extensions (RSVP-TE) [2], [3]. RSVP-TE is a destination-initiated reservation protocol, that involves a signaling from the source to the destination node of the lightpath (referred to as forward signaling) and viceversa (backward signaling). After selecting a route based on network topology information, RSVP-TE protocol checks resource (i.e., wavelength) availability at each intermediate node of the route during the forward signaling and, then, it effectively reserves a wavelength on each link of the route during the backward signaling.

In absence of wavelength conversion capabilities, an RSVP-TE protocol instance may be blocked during the forward signaling (i.e., forward blocking) due to the unavailability of a common wavelength on all the route links, or during the backward signaling (i.e., backward blocking) due to resource contentions [4]. Resource contention is caused by the concurrent attempts of two or more signaling instances to reserve the same wavelength on a link.

The RSVP-TE protocol is responsible for provisioning the requested lightpaths. When the network is in working condition, the backward blocking becomes the predominant reason of blocking, if the lightpath arrival process is highly dynamic or if the traffic offered to the network is low [4], [5]. Moreover, when the network is in failure condition, a single fault (e.g., link fault) simultaneously affects a number of lightpaths and a RSVP-TE protocol instance is activated for recovering each disrupted lightpath [6]. In this case, the backward blocking may also dominate over the forward blocking due to the numerous concurrent instances that contend the available (i.e., not reserved) wavelengths.

The probability of backward blocking, or backward blocking probability, can be reduced in two different ways: by reducing the probability that RSVP-TE instances may contend the wavelengths or by selecting and reserving disjoint wavelengths for concurrent reservation attempts along the same links. The former solution can be achieved, for example, by over-reserving the wavelengths during the forward signaling, so that the backward signaling may only release non-selected wavelengths [7]. However, the over-reservation unnecessarily increases the forward blocking. The latter solution is considered by few different schemes [5], [6], [8], [9]. The work in [8] presents a wavelength-weighted scheme, compliant with RSVP-TE signaling, that reduces the backward blocking during provisioning. However, the scheme is suitable only during working conditions, since the wavelength selection is based on weights collected and applied during the steady state. The reservation collision avoidance scheme (RCA) introduced in [5], [6] can be applied to RSVP-TE message exchange for 
selecting wavelengths during lightpath provisioning as well as restoration. Wavelength selection can also be performed using a circular wavelength-list for reducing the backward blocking during provisioning, as proposed in [9]. The scheme, however, requires an additional signaling message with respect to RSVP-TE protocol.

In addition, it is worth to mention other relevant schemes for optimal wavelength selection in dynamic WDM networks, such as [10] and [11]. In particular, [10] proposes a scheme to be applied to a forward reservation protocol during resource provisioning. [11] proposes a scheme for a forward reservation protocol, based on message broadcasting, to be applied during restoration. However, both schemes are specifically designed for forward reservation protocols, instead of a backward reservation protocol like RSVP-TE.

This paper proposes two schemes that aim at minimizing the backward blocking probability in working and failure conditions of dynamic distributed wavelength-routed networks with wavelength continuity constraint. The schemes, named suggested label (SL) and suggested vector (SV), use the standard Suggested Label object [3], to provide the destination with the identification of the wavelength to be preferably reserved, for the requested lightpath. Both schemes keep track of potential contentions as proposed in [5], [6], i.e., by including an additional one-bit object, called Contention Detection object, in the control message sent in the forward direction (i.e., Path message). In addition to the above mentioned objects, the more sophisticated scheme, i.e., SV scheme, keeps record of the previously suggested labels i.e., by including the Suggested Vector object [12] in the Path message. Once the standard object (i.e., Suggested Label object) and the proposed objects (i.e., Contention Detection object and Suggested Vector object) are included in the RSVP-TE control messages, the destination node is provided with an indication of potential contentions (in the Contention Detection object) and a suggestion of the label (in the Suggested Label object) to be preferably reserved and, optionally, a set of label preference weights (in the Suggested Vector object). For this reason, the schemes are referred to as label preference (LP) schemes.

The strength of the proposed schemes lays in the ability to differentiate the wavelength selection strategy depending on the presence of potential contentions in intermediate nodes. A comprehensive study of the performance of the LP schemes using different wavelength selection strategies has been carried out in a ring and in a mesh topology during working and failure network conditions. The simulation results are then compared against other known schemes. The results show that the proposed LP schemes are effective in reducing the backward blocking probability, without affecting the forward blocking probability, and that they outperform other known schemes under a variety of network loads. As a result, the use of LP schemes allows to achieve a reduction of the overall blocking probability in working condition and an increase of the overall network reliability upon failure occurrence. Finally, the scheme based on the Suggested Vector object trades the use of an additional object for a better performance.

\section{LIGHTPATH SETUP IN GMPLS NETWORKS}

The considered scenario is a wavelength-routed optical network. The physical topology of the network can be represented by a graph $G(N, L)$, where $N$ is the set of nodes and $L$ is the set of bidirectional links. A link exists in $G(N, L)$ if a fiber, supporting $W$ wavelengths, interconnects a node pair. Each node is equipped with an all-optical cross-connect, without wavelength conversion capabilities, for switching wavelength-continuous lightpaths.

GMPLS control plane manages the network through out-ofband signaling. It is assumed that the control plane topology coincides with physical topology graph, $G(N, L)$. In each node, GMPLS control plane maintains a database with the network topology information and a database with the wavelength availability information for each outgoing link.

In working condition, the control plane is responsible for lightpath provisioning. In failure condition, the control plane is responsible for fault advertisement and lightpath restoration. Fault advertisement is initiated by the node detecting the failed element (e.g., the downstream node of the failed link in case of link failure) and achieved by informing all the source nodes of the disrupted lightpaths about the type of detected error and the faulted element identifier, with RSVP-TE Notify messages defined in [3]. Upon reception of the Notify, each node updates the network topology information and initiates the lightpath restoration.

Upon arrival of a lightpath provisioning or restoration request, the source computes the path to destination, based on the topology information stored in the database, and initiates the signaling procedure utilizing RSVP-TE. The RSVP-TE is based on two types of messages: the Path message sent from the source toward the destination (forward direction) and the Resv sent from the destination toward the source (backward direction).

The Path message includes the following objects: the Explicit Route object [2] and the Label Set object [3]. The Explicit Route object specifies the route, computed at source node, that the Path and the Resv messages should follow. The Label Set object is implemented as an array of labels identifying the wavelengths that can be used for the requested lightpath.

Initially, the source creates the Label Set object with the list of wavelengths that are available on the outgoing link indicated in the Explicit Route object. Upon reception of the Path message, each intermediate node updates the Label Set object by intersecting the received Label Set with the set of wavelengths available on the outgoing link. The updated Path message is then forwarded to the next hop (i.e., next node in the Explicit Route Object). When the Path message reaches the destination, the Label Set contains the wavelengths, that are available on the end-to-end path, thus satisfying the wavelength-continuity constraint.

Upon reception of the Path message, the destination selects one of the available wavelengths contained in the Label Set object, according to a tie breaking policy. A Resv message is sent by the destination in the backward direction, to reserve the selected wavelength on each link of the route and to set the optical cross-connect switches at each node. Upon reception of the Resv message, each intermediate node locks the selected wavelength, updates the list of available wavelengths on its outgoing link, and forwards the Resv message to the next node toward the source. Once the Resv message reaches the source, the lightpath is established and data transmission can take place. 
In the described scenario, two are the cases of early termination (i.e., blocking) of a reservation protocol instance, that cause an unsuccessful lightpath establishment:

- Forward Blocking: the propagation of the Path message in the forward direction is interrupted, when the intersection between the Label Set object and the set of wavelengths available on the next hop is empty. An error message (PathErr) is generated and sent toward the source, to inform that no wavelengths, satisfying the wavelengthcontinuity constraint, are available on the selected path.

- Backward Blocking: the propagation of the Resv message in the backward direction is interrupted, when an attempt to reserve an already reserved wavelength is made (i.e., contending reservation attempt). An error message (PathErr) is generated and sent toward the source to report the blocking, while another error message (ResvErr) is generated and sent toward the destination to free the already reserved wavelength on the various links. Backward blocking is due to outdated information: the selected wavelength, that the Path message detected as available, has been reserved on a link by some other ongoing reservation attempts and the wavelength state changed by the time the Resv message arrived on that link.

For tearing down a lightpath, a PathTear (or, alternatively, a ResvTear) message is sent by the source (destination) in the forward (backward) direction, to release the wavelength used by the lightpath.

The specifications for RSVP-TE protocol are limited to the structure of the signaling messages and the objects within. The algorithms used to compute the object content are a local matter of each network node, i.e., no standard behavior is needed because they may aim at different purposes.

\section{LABEL PREFERENCE SCHEMES}

This section presents the two proposed schemes, referred to as label preference (LP) schemes. By using these schemes, the RSVP-TE manages protocol objects for providing the destination node with a label, that identifies the wavelength to select for provisioning or restoring lightpaths. The aim of the schemes is to reduce the backward blocking probability, by minimizing the probability of wavelength contentions among concurrent reservation attempts, without impacting the forward blocking probability.

After a brief explanation of the Path message objects and the database that are required by the LP schemes, the proposed schemes will be detailed.

\section{A. LP Schemes Objects and Databases}

The proposed LP schemes require three additional objects in the Path message: the Suggested Label (SL) object, the Contention Detection (CD) object, and the Suggested Vector (SV) object. The Suggested Label object is an optional object defined by RSVP-TE in [3] and it is used here to identify the suggested wavelength. Contention Detection and Suggested Vector objects are here defined and proposed for inclusion in the Path message.

The Contention Detection object is one bit flag proposed in [5], [6] to indicate whether the current reservation attempt may experience (i.e., CD bit set to True) a backward blocking or not (i.e., CD bit set to False). The Suggested Vector object is a vector containing weights, i.e., preference levels, for each label of the Label Set object, as proposed in [12].

Each node is required to maintain a database of path state blocks (PSBs) and a database of reservation state blocks (RSBs) [13]. A PSB stores the information contained in each received Path message (i.e., incoming Path message), including the carried objects. In addition, the PSB is required to store the Label Set object and the Suggested Label object computed for each Path message to be forwarded to the next hop (i.e., outgoing Path message). An RSB stores the information contained in each received Resv message.

The information in a PSB (RSB) is valid for the period of time elapsing between the passage of the Path (Resv) message and the passage of the corresponding PathTear or ResvTear message (in case of successful reservation attempt), or the passage of the corresponding PathErr or ResvErr message (in case of unsuccessful reservation attempt).

\section{B. The Suggested Label (SL) Scheme}

This section describes the LP scheme that is based on the Suggested Label object and is thus referred to as suggested label (SL) scheme. The SL scheme uses the Contention Detection and the Suggested Label objects. With this scheme, RSVP-TE protocol provides the destination with a single preferred label, the one indicated in the Suggested Label object.

Upon reception of a lightpath provisioning or restoration request, the source node initializes the Contention Detection and the Suggested Label objects as follows:

- Initialization of the Contention Detection (CD) object. CD bit is set to False.

- Initialization of the Suggested Label object. For lightpath provisioning, the suggested label is chosen according to the label selection strategy considered for RSVP-TE (e.g., first-fit). For lightpath restoration, the suggested label is chosen according to a strategy that maps in a biunique way the wavelength used by the lightpath before failure into another (or eventually the same) wavelength, among those that are less used during provisioning. This criterion guarantees that: 1) thanks to the biuniqueness of the mapping, the initially selected suggested label is different from those of the other disrupted lightpaths (as it was in working condition) and 2) the suggested label has a higher probability of being available. For example, if during provisioning the suggested labels are initially selected using a first-fit strategy, during restoration the suggested label, $\lambda_{r}$, for a disrupted lightpath can be selected as

$$
\lambda_{r}=W+1-\lambda_{p}
$$

where $W$ is the number of wavelengths on each link and $\lambda_{p} \in\{1,2, \ldots, W\}$ indicates the wavelength that supported the lightpath before failure.

The source node and each intermediate node along the path have the possibility to modify the Contention Detection object and the Suggested Label object, based on the Contention Detection object and on the information stored in the local PSB and RSB databases. The operations performed by the nodes on 


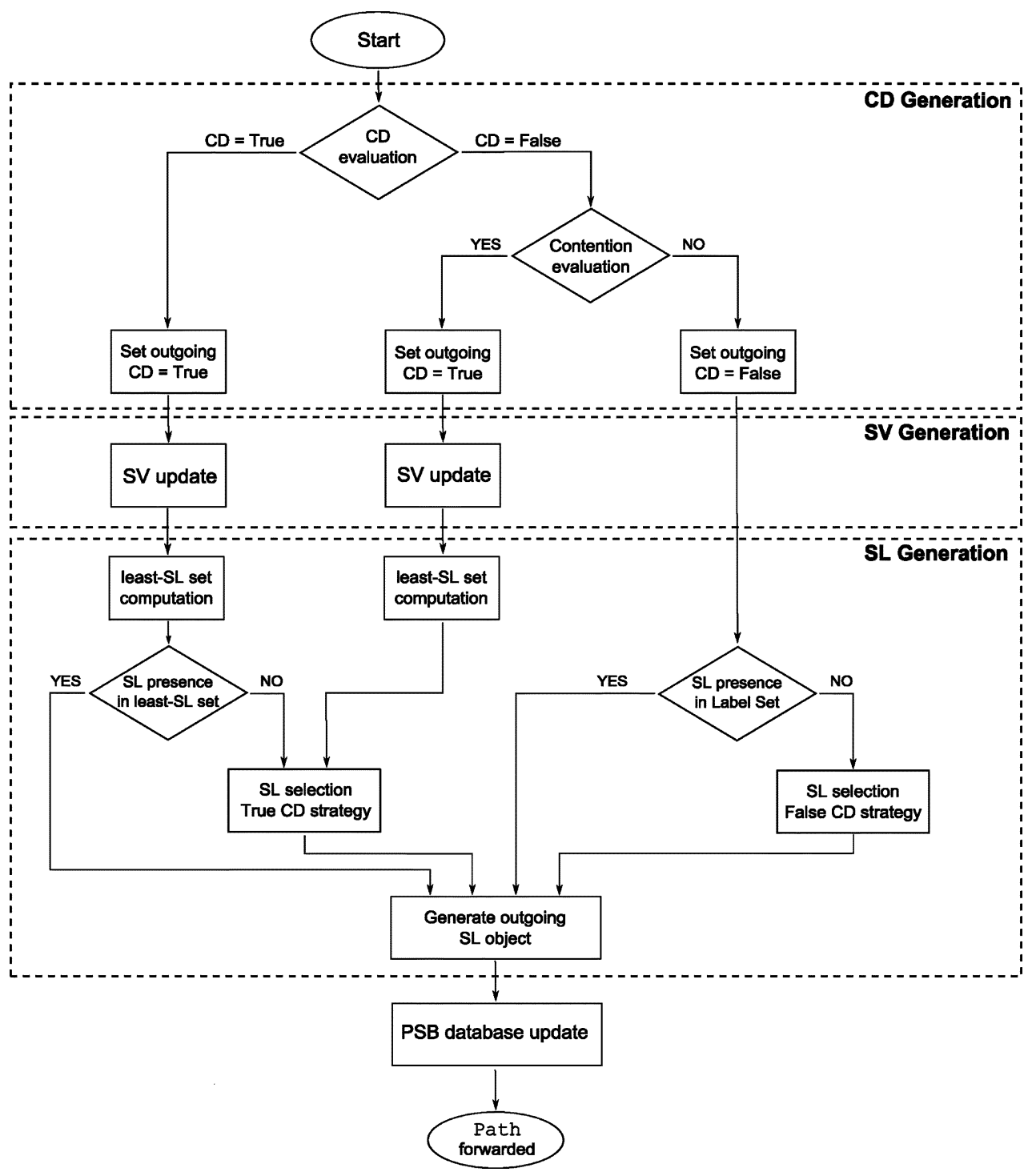

Fig. 1. Flowchart of the Path message processing operations for the proposed LP schemes (Suggested Vector update box is executed only in SV scheme).

these objects are described next and their sequence is sketched in Fig. 1. (Notice that, for the SL scheme, the Suggested Vector generation box in Fig. 1 is not executed.)

Upon initialization at the source or upon reception of a Path message at an intermediate node, the node computes the outgoing Label Set as described in Section II and, then, it performs the following steps:

- Generation of the Contention Detection (CD) object. To generate the outgoing $\mathrm{CD}$ object, the following operations are performed:

- Evaluation of the incoming Contention Detection object. The value of the incoming $\mathrm{CD}$ bit is checked: if True, the outgoing CD bit is set to True; if False, the contention evaluation operation is performed as explained next.

- Contention evaluation. Let us define a reservation attempt as potentially contending with the ongoing reservation if: 1) it has a PSB in the node and passes on the same next hop of the incoming Path message, and 2) it does not have a RSB (i.e., a Resv backward message for such attempt has not been received yet). The outgoing $\mathrm{CD}$ bit is set to False in case of empty intersection between the outgoing Label Set object and any Label Set of the potentially contending reservations, stored in the PSBs. Otherwise, the CD bit is set to True.

- Generation of the Suggested Label (SL) object. To generate the outgoing SL object, the following operations are performed:

- Computation of the least-SL set. The least-SL set is defined as a subset of the outgoing Label Set, whose labels are present the minimum number of times, in the outgoing SL objects of the potentially contending reservations (stored in the node PSBs).

- Presence of the SL object in least-SL set. When the incoming CD bit is True, the presence of the incoming Suggested Label object is checked in the least-SL set. 
If present, the outgoing SL object is the same as the incoming one. If absent, it is computed following the $S L$ selection operation detailed below.

- Presence of the SL object in Label Set object. When the outgoing CD bit is False, the presence of the SL object in the Label Set is checked. If present, the outgoing SL object is the same as the incoming one. If absent, the outgoing SL object is computed following the SL selection operation detailed next.

- SL selection. The Suggest Label is selected by applying a default strategy when the outgoing CD bit is False (i.e., False CD strategy) and a distinct strategy when the outgoing CD bit is true (i.e., True CD strategy). Options for False and True CD strategies may include, for example, first-fit, last-fit and random selection and are evaluated in Section IV. The set of labels on which the strategies are applied is the least-SL set or the Label Set, depending on whether the outgoing CD bit is True or False, respectively.

Once the label to be suggested has been selected, the outgoing SL object is generated.

- PSB database update. A new PSB is added in the database containing the information of the incoming Path message and the outgoing Label Set and Suggested Label objects;

- Path message forwarding. The outgoing Path message is generated with the SL object and the CD object, and it is forwarded to the next hop.

Upon reception of a Path message, the destination generates a Resv message for the reservation of the wavelength indicated by the Suggested Label object in the incoming Path message.

In the backward direction, each intermediate node receiving a Resv message reserves the selected wavelength, adds a RSB in the database, and forwards the message to the next node toward the source.

\section{The Suggested Vector (SV) Scheme}

This section describes the label preference scheme, based on the Suggested Vector object. The scheme is, thus, referred as suggested vector $(S V)$ scheme. The SV scheme uses the Contention Detection, the Suggested Label and the Suggested Vector objects. As for the SL scheme, when using SV scheme, the RSVP-TE protocol provides the destination with a preferred label, as indicated in the Suggested Label object.

The initialization procedure at the source is as follows:

- Initialization of the Contention Detection (CD) object. Same as SL scheme (Section III-B).

- Initialization of the Suggested Label object. Same as SL scheme (Section III-B).

- Initialization of the Suggested Vector object. All the entries of the Suggested Vector object are initialized to the parametric value $\gamma$, except during restoration when the specific entry corresponding to the suggested label [i.e., $\lambda_{r}$ in (1)] is initialized to zero.

The source node and each intermediate node have the possibility to modify the objects, based on the Contention Detection object, the Suggested Label object, and the information stored in the PSBs and RSBs. In particular, the weights in the Suggested Vector objects are updated to keep record of the labels suggested by the upstream nodes and the potential contentions on the corresponding wavelengths.

Using the RSVP-TE signaling, the operations for handling the Suggested Label object are similar to those defined for the SL scheme (Section III-B) with the additional operation for Suggested Vector generation, as sketched in Fig. 1. The operations are described next.

Upon initialization at the source or upon reception of a Path message at an intermediate node, the node computes the outgoing Label Set as described for the RSVP-TE protocol in Section II and, then, it performs the following steps:

- Generation of the Contention Detection (CD) object. Same as SL scheme (Section III-B).

- Generation of the Suggested Vector (SV) object. For each label $l$ in the outgoing Label Set, a weight $W_{o}(l)$ is calculated as

$$
W_{o}(l)=W_{i}(l)+\alpha \cdot n_{\mathrm{LS}}(l)+\beta \cdot n_{\mathrm{SL}}(l)
$$

where $W_{i}(l)$ is the weight associated with label $l$ in the incoming Suggested Vector object, $n_{\mathrm{LS}}(l)$ is the number of times that label $l$ is present in the outgoing Label Set objects of the potentially contending reservations, stored in PSBs, and $n_{\mathrm{SL}}(l)$ is the number of times that label $l$ is present in the outgoing Suggested Label objects of the potentially contending reservations, stored in PSBs. The parameters $\alpha, \beta, \gamma>0$ can be tuned so that a minimum weight is assigned to the wavelength to be selected by the destination node. During provisioning, the following selection criterion is proposed: $p_{1}$ ) select the wavelength that the potentially contending reservations chose as suggested label the minimum number of times and, in case of ties among them, select the wavelength that the potentially contending reservations included in the Label Set objects the minimum number of times. To apply such criterion, it is necessary to tune the parameter values in (2) such that: $p_{1}$ ) $\beta \gg \alpha$. During restoration, the following selection criteria are proposed: $\left.r_{1}\right)$ select the wavelength suggested by the source node (i.e., $\lambda_{r}$ in (2)) if it is chosen as suggested label the minimum number of times by other potentially contending reservations, otherwise $r_{2}$ ) as $p_{1}$. To apply such criteria, it is necessary to tune the parameter values in (2) such that: $\left.\left.r_{1}\right) \gamma \gg \alpha, r_{2}\right)$ and $\beta-\gamma \gg \alpha$.

With the calculated weights, the Suggested Vector object is, then, generated.

- Generation of the Suggested Label (SL) object. Same as SL scheme (Section III-B). However, in this case, the least-SL set is computed based on the weights stored in the Suggested Vector object, as explained below.

- Computation of the least-SL set. The least-SL set is generated by including the labels present in the outgoing Label Set and with the minimum weight in the outgoing Suggested Vector object.

- PSB Database Update. Same as SL scheme (Section III-B). 


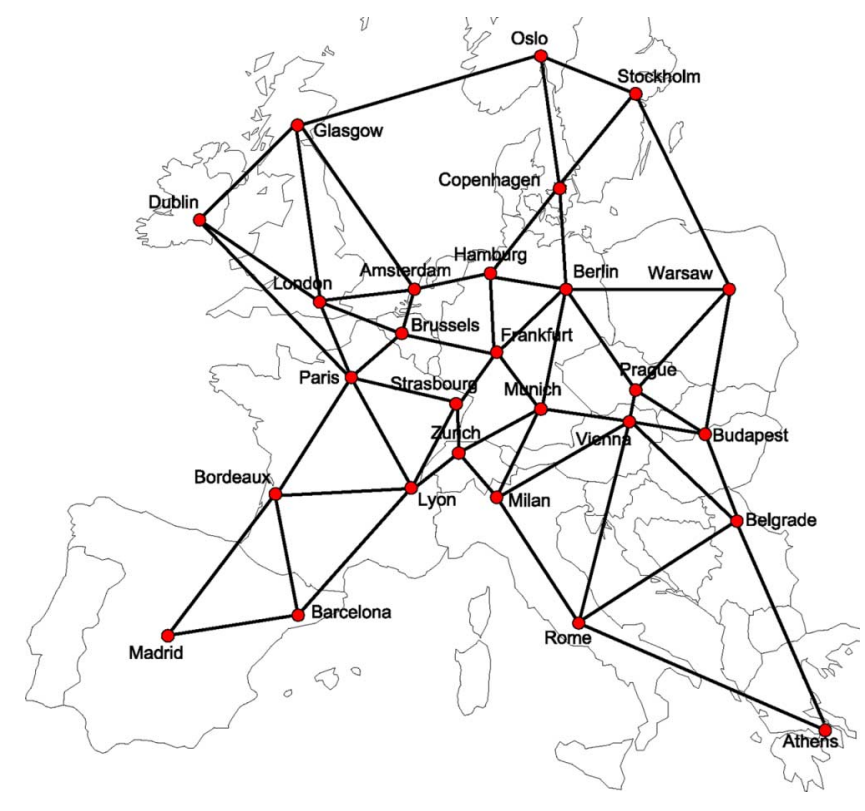

Fig. 2. Pan-European network topology.

- Path message forwarding. The outgoing Path message is generated with the Contention Detection object, the Suggested Label object, and the Suggested Vector object and it is forwarded to the next hop.

The operations to be performed at the destination and in the backward direction are the same as in the SL scheme, described in Section III-B.

\section{CASE Study}

The performance study of label preference (LP) schemes, i.e., SL and SV schemes, has been carried out on two network topologies: the Pan-European network with 27 nodes and 55 links shown in Fig. 2 and a ring topology with 27 nodes. Each link of the two network topologies is bidirectional and carries $W=32$ wavelengths per each direction.

Lightpath provisioning requests are dynamically generated following a Poisson process and uniformly distributed among the source-destination pairs. The inter-arrival time and holding time of the lightpath requests are exponentially distributed with an average of $1 / \lambda$ and $1 / \mu$ seconds, respectively. The load offered to the network in working state condition is, therefore, expressed in Erlangs as the ratio $\lambda / \mu$. Provisioned and restored lightpaths are routed along the shortest path (in terms of number of hops) on the network topology. In the case of multiple shortest paths, one of the shortest paths is randomly selected each time. Link lengths are proportional to the geographical distance of the nodes. Processing time of the packets is considered negligible compared to the optical propagation and transmission time. In the SV scheme, the parameters $\alpha, \beta, \gamma$ are set to 1 and 1000 and 500, respectively, to satisfy the conditions: $\beta \gg \alpha, \gamma \gg \alpha$, and $\beta-\gamma \gg \alpha$, discussed in Section III-C.

The results are obtained by running a custom-built $\mathrm{C}++$ eventdriven simulator [14] and are collected until the confidence interval of 5\% at $95 \%$ confidence level or the maximum number of independent trials (i.e., $10^{3}$ in the provisioning phase, and $10^{4}$ in the restoration phase) is reached. All results are then plotted with the confidence interval at $95 \%$ confidence level.

First-fit, last-fit, and random wavelength assignment strategies have been considered for the various schemes. The best performing combinations of strategies are compared next.

\section{A. LP Schemes Performance in Provisioning}

When the network operates in working condition (i.e., in the provisioning phase), the performance of the proposed LP schemes is evaluated in terms of blocking probability. The blocking probability is defined as the probability that a reservation attempt is blocked in either the forward (i.e., forward blocking) or the backward (i.e., backward blocking) direction.

Each plot details the forward and backward blocking components and their sum (i.e., the overall blocking) in the considered scenarios. Based on the results on lightpath provisioning presented in [5], [6], the following suggested label selection strategies (i.e., True CD strategy and False CD strategy in Fig. 1) are chosen for both LP schemes:

- first-fit (FF) is used as False CD strategy;

- last-fit (LF) is used as True CD strategy.

Using these strategies, the SL scheme is indicated as SL-FF/LF. The SV scheme is indicated as SV-FF/LF. LP schemes are compared against:

- RSVP-FF and RSVP-RD: the standard RSVP-TE protocol with a first-fit (FF) strategy and a random (RD) strategy, respectively, applied to the Label Set object by the destination;

- CD-FF/LF: the scheme based on the use of the Contention Detection object only (i.e., without Suggested Label and Suggested Vector objects), as proposed in [5], [6]. At the destination, the wavelength is selected according to the first-fit or last-fit strategy on the incoming Label Set object, depending on whether the incoming $\mathrm{CD}$ bit is False or True, respectively;

- CWL: the scheme introduced in [9], that is based on the Circular Wavelength List (CWL) object. In this scheme the source randomly selects a suggested label in the CWL. At an intermediate node, if the suggested label is unavailable or is already suggested by another concurrent reservation attempt, another suggested label is selected using a first-fit strategy applied to CWL. In addition to RSVP-TE message exchange, a novel control message is then sent to the source by each intermediate node that modifies the Suggested Label object.

Figs. 3 and 4 show the blocking probabilities as a function of the network load (when the mean holding time is $1 / \mu=60 \mathrm{~s}$ ) for the Pan-European and the ring topology, respectively. For clarity, Figs. 3(a) and 4(a) refer to RSVP-FF, RSVP-RD, and CWL schemes, while Figs. 3(b) and 4(b) refer to CD-FF/LF, SL-FF/LF, and SV-FF/LF schemes. The offered network load is varied by changing the mean inter-arrival time. The figures demonstrate that, for low network loads, the overall blocking is driven by the backward blocking.

Moreover, the comparison of RSVP-FF and RSVP-RD schemes [Figs. 3(a) and 4(a)] reveals that the random strategy achieves a backward blocking lower than first-fit strategy and it is therefore more effective in reducing contentions. However, 


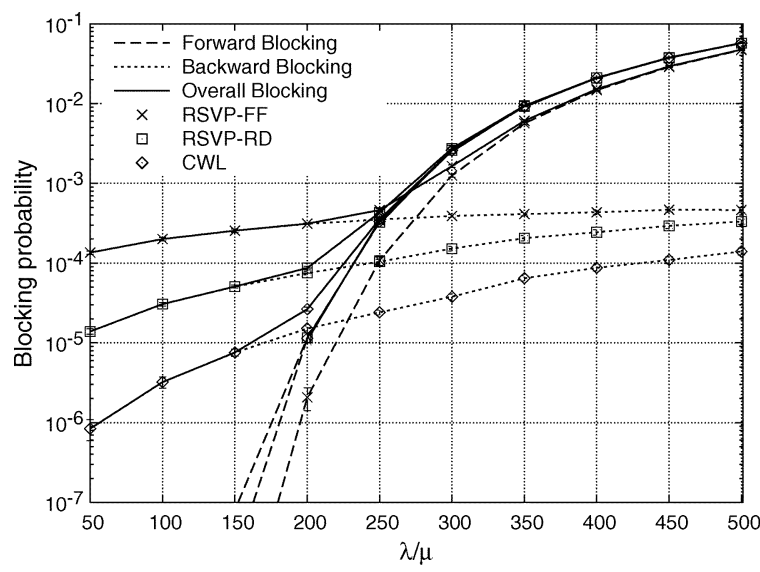

(a)

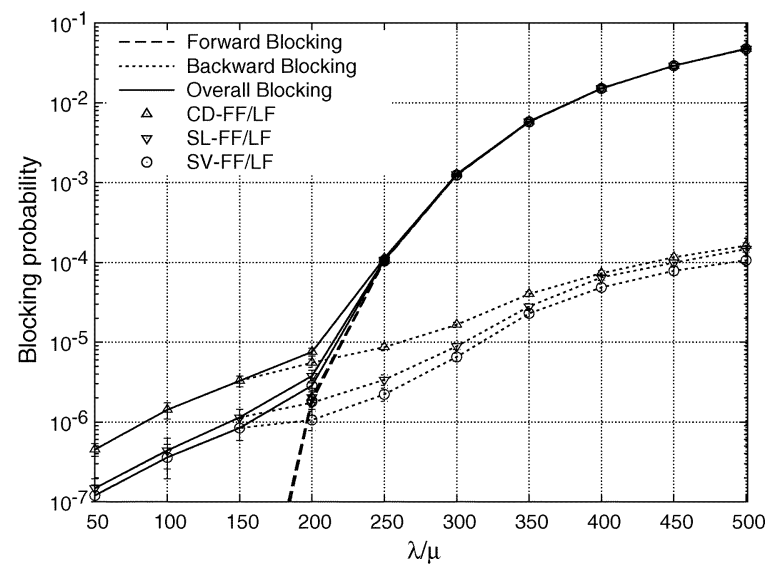

(b)

Fig. 3. Provisioning in the Pan-European network: blocking probabilities versus network load (in Erlangs), $1 / \mu=60 \mathrm{~s}$. (a) RSVP-FF, RSVP-RD, and CWL schemes. (b) CD-FF/LF, SL-FF/LF, and SV-FF/LF schemes.

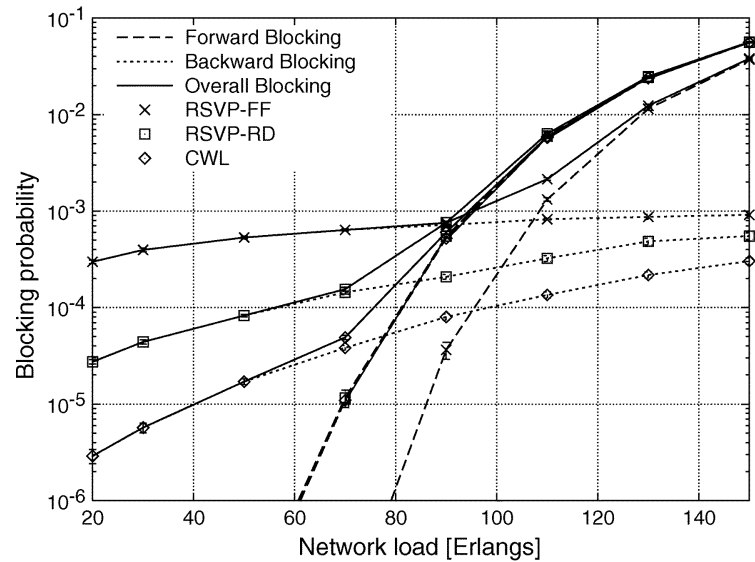

(a)

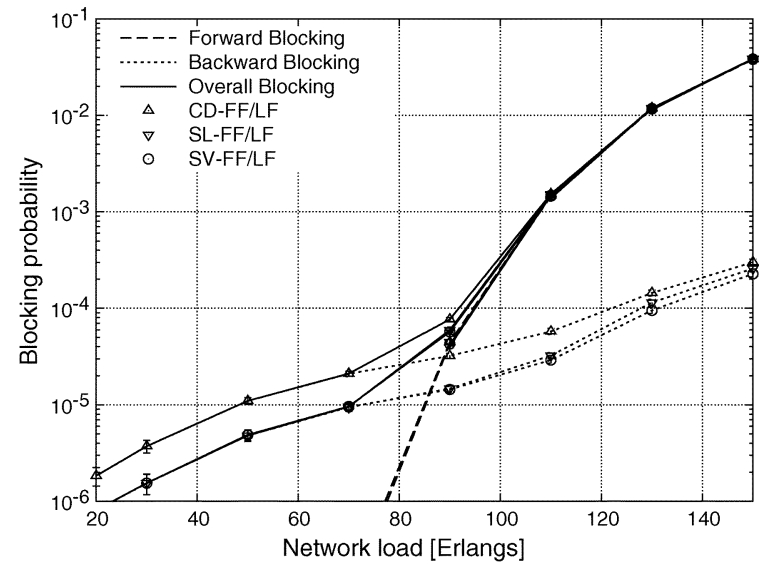

(b)

Fig. 4. Provisioning in the ring network: blocking probabilities versus network load (in Erlangs), $1 / \mu=60 \mathrm{~s}$. (a) RSVP-FF, RSVP-RD, and CWL schemes. (b) CD-FF/LF, SL-FF/LF, and SV-FF/LF schemes.

the first-fit strategy achieves a lower forward blocking, thanks to its ability to pack the lightpaths on the wavelengths. The figure details also the performance of the CWL scheme. As shown in [9], the use of the CWL object and the additional signaling messages of CWL scheme are able to further reduce the backward blocking of RSVP-RD scheme. However, the forward blocking of CWL scheme is as high as in RSVP-RD scheme, since the selection of the suggested label at the sources is performed randomly.

The CD-FF/LF scheme [Figs. 3(b) and 4(b)] proposed in [5] and [6] combines the benefits of the two traditional strategies, i.e., first-fit and last-fit, during the label selection performed at the destination. On the one hand, by applying first-fit strategy when CD bit is False, the CD-FF/LF scheme exploits the resources at best as in RSVP-FF scheme and, thus, achieves similar forward blocking. On the other hand, by applying a different selection strategy (i.e., last-fit) when the CD bit is True, CD-FF/LF scheme improves the backward blocking with respect to both RSVP-RD and CWL schemes.

In addition, Figs. 3(b) and 4(b) show that the proposed SL and SV schemes outperform the CD-FF/LF scheme, by further reducing the backward blocking, while maintaining the same level of forward blocking as in RSVP-FF and CD-FF/LF schemes. In particular, the SL-FF/LF scheme combats the backward blocking, by selecting the suggested label in the intermediate nodes, depending on the ongoing reservation attempts. The SV-FF/LF scheme better opposes the backward blocking, by assigning weights to the labels in each intermediate node.

The figures also show that the performance achieved in the Pan-European [Figs. 3(a) and 3(b)] and in the ring network [Figs. 4(a) and 4(b)] are comparable. Therefore, also due to space constraints, only results on the Pan-European topology are shown in the following.

Fig. 5 shows the blocking probabilities suffered by the PanEuropean network as a function of the mean inter-arrival time, when the mean holding time is varied to achieve a fixed network load of $\lambda / \mu=250$ Erlangs. Both figures demonstrate that the backward component of the blocking probability dominates for low values of the mean inter-arrival time, i.e., for inter-arrival times lower than or equal to the mean round-trip time (about $12 \mathrm{~ms}$ for the considered topology).

Fig. 5(b) confirms that the CD-FF/LF scheme and the proposed SL and SV schemes achieve a forward blocking 


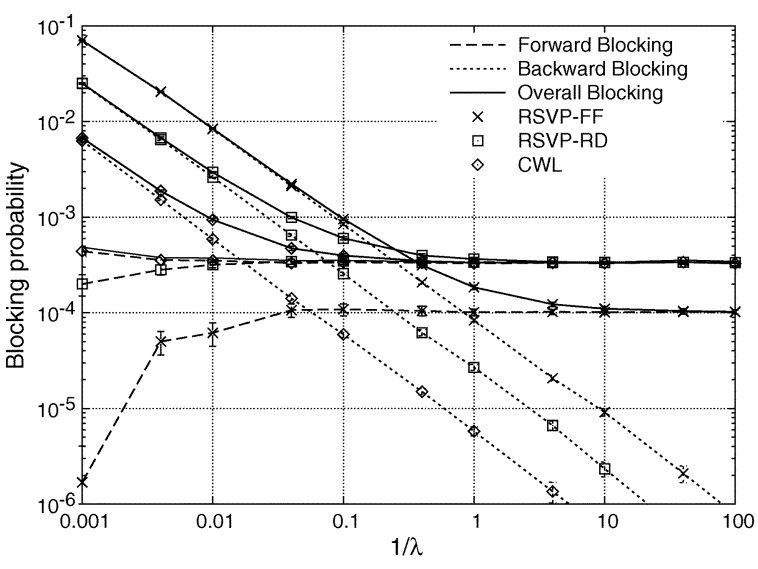

(a)

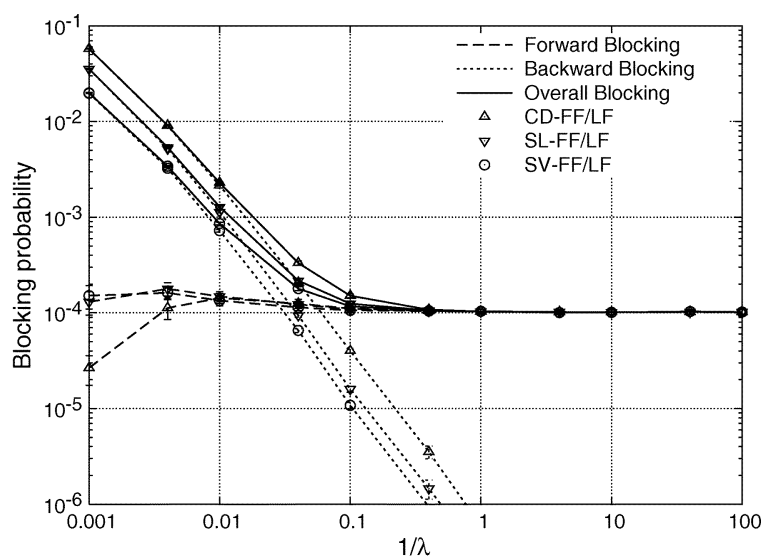

(b)

Fig. 5. Provisioning in the Pan-European network: blocking probabilities versus mean inter-arrival time (in seconds), $\lambda / \mu=250$ Erlangs. (a) RSVP-FF, RSVP-RD, and CWL schemes. (b) CD-FF/LF, SL-FF/LF, and SV-FF/LF schemes.

probability as low as that of RSVP-FF scheme, while the CWL scheme achieves a forward blocking probability as high as that of RSVP-RD scheme [see Fig. 5(a)].

For inter-arrival times higher than $0.1 \mathrm{~s}$, the proposed LP schemes with FF/LF strategies are very effective in reducing resource contentions. The reason is that, for these values of interarrival time, usually only two concurrent reservations may contend the same wavelength and are assigned two distinct wavelengths by the LP schemes with FF/LF strategies, i.e., a lowindexed and a high-indexed wavelength. However, for lower values of inter-arrival time, the CWL scheme guarantees the best performance in terms of backward blocking [Fig. 5(b)]. The reason is that, in highly dynamic traffic scenario, several concurrent reservation attempts may contend the same wavelength, and thus blocking is reduced by the random selection strategy used in the CWL scheme.

\section{B. LP Schemes Performance During Restoration}

The performance of the proposed LP schemes during restoration is evaluated in terms of restoration blocking probability. The restoration blocking probability is defined as the probability that a restoration attempt is blocked in either the forward direction (i.e., forward blocking) or the backward direction (i.e., backward blocking). It is calculated as the ratio between the number of unsuccessfully restored lightpaths and the number of lightpaths affected by the fault.

Source nodes initialize the Suggested Label object according to (1). Moreover, based on the better performance of CD-FF/RD than CD-FF/LF [5], [6] during highly dynamic lightpath provisioning, the following suggested label selection strategies (i.e., True CD strategy and False CD strategy in Fig. 1) are chosen:

- first-fit (FF) is used as False CD strategy;

- random (RD) is used as True CD strategy.

During restoration, FF/RD strategies are presented instead of $\mathrm{FF} / \mathrm{LF}$ as they achieve better performance. The reason is that, during restoration, a resource contention typically occurs among a number (i.e., more than two) of reservation instances, so the use of RD as True CD strategy allows to (randomly) select different wavelengths (instead of the same high-indexed wavelength selected by LF strategy) for the several contending instances tagged with True CD bit.

Using these strategies, the SL scheme is indicated as SL-FF/RD and the SV scheme is indicated as SV-FF/RD. The performance of both LP schemes are compared against RSVP-FF, RSVP-RD, CD-FF/RD, and CWL, as described in Section IV-A.

In all the schemes, for a given network load, the network is provisioned with lightpaths using first-fit wavelength assignment (i.e., RSVP-FF scheme). Once steady state conditions are reached, the simulator generates single-link failure events that are randomly distributed on the network links. After each link failure, performance statistics are collected.

Fig. 6(a) plots the overall blocking probability versus network load, when the mean inter-arrival time is $1 / \lambda=0.1 \mathrm{~s}$. The figure demonstrates that even in restoration the LP schemes are able to reduce the overall blocking probability with respect to the other schemes. In particular, it shows that the SV-FF/RD scheme strongly outperforms the other schemes, especially for low network load when most of the disrupted lightpaths are restored, thanks to the suggested label computed by the source node as indicated in (1).

Fig. 6(b) details the backward and the forward restoration blocking probabilities. The figure clearly shows that the main contribution to the blocking probability is given by the backward blocking for all the schemes. Moreover, as the network load increases, the forward blocking becomes more important. The forward blocking experienced during restoration is approximately the same for all the schemes. The reason is that the forward blocking is due to the lack of network resources and thus it is mainly determined by how wavelengths are allocated during provisioning (i.e., RSVP-FF for all schemes).

Fig. 6(b) shows, also, that the LP schemes achieve a backward blocking probability lower than other schemes. Among the LP schemes, SV-FF/RD scheme performs better than SL-FF/RD scheme due to the possibility of keeping a weighted record of the previously suggested labels. For very high network loads, the improvement of the backward blocking probability decreases 


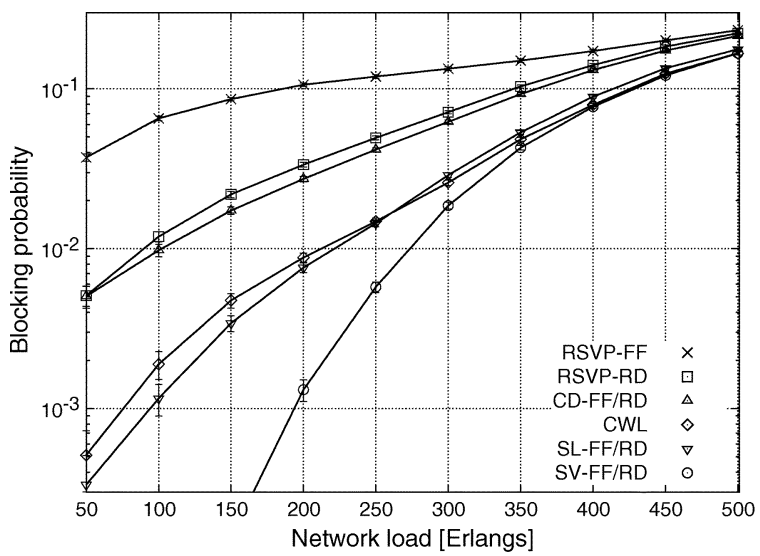

(a)

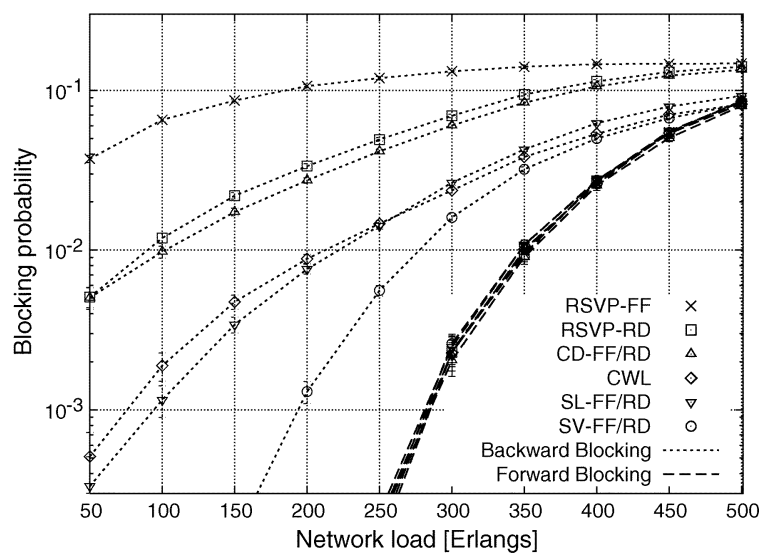

(b)

Fig. 6. Restoration in the Pan-European network: blocking probabilities versus network load (in Erlangs), $1 / \lambda=0.1 \mathrm{~s}$. (a) Overall restoration blocking probability. (b) Forward and backward restoration blocking probability.

since the label preference schemes cannot easily operate with only few wavelengths available for the restoration paths.

\section{CONCLUSION}

This paper investigated wavelength-routed networks with wavelength continuity constraint and with a GMPLS distributed control plane without knowledge of the wavelength availability. In this scenario, the RSVP-TE protocol is used to reserve wavelengths for (re)establishing lightpaths. The paper showed that, in highly dynamic traffic scenario as well as during restoration, the main reason of the unsuccessful wavelength reservation (i.e., blocking) is due to outdated and inaccurate information on the wavelength availability (i.e., backward blocking) caused by multiple concurrent reservations, rather than the lack of resources (i.e., forwarding blocking).

To alleviate this problem, the paper proposed two LP schemes to be applied to RSVP-TE protocol, with the aim of optimizing the wavelength selection. The schemes, referred to as SL and SV schemes, provide the destination with a label, identifying the wavelength to reserve. The strategy for selecting the preferred label at intermediate and destination nodes aims at assigning disjoint wavelengths to concurrent reservation attempts contending the resources.

Simulation results demonstrated that LP schemes are effective in reducing the backward blocking during lightpath provisioning and restoration, without negatively impacting the forward blocking. Performance comparisons attest that, by keeping a record of the previously suggested labels through a set of weights, SV scheme obtains a lower restoration blocking probability compared to the memory-less scheme (i.e., SL scheme). Finally, the benefits achievable by the proposed schemes have minimal impact on the complexity of the RSVP-TE protocol as the schemes are compliant with RSVP-TE behavior and need only one or two additional objects to be included in the standard messages.

\section{REFERENCES}

[1] E. Mannie, Generalized Multi-Protocol Label Switching (GMPLS) Architecture, IETF, RFC 3945, Oct. 2004.

[2] D. Awduche, L. Berger, D. Gan, T. Li, V. Srinivasan, and G. Swallow, RSVP-TE: Extensions to RSVP for LSP Tunnels, IETF, RFC 3209, Dec. 2001.

[3] L. Berger, Generalized Multi-Protocol Label Switching (GMPLS) Signaling Resource Reservation Protocol-Traffic Engineering (RSVP-TE) Extensions IETF, RFC 3473, Jan. 2003.

[4] K. Lu, G. Xiao, and I. Chlamtac, "Analysis of blocking probability for distributed lightpath establishment in WDM optical networks," IEEE/ACM Trans. Netw., vol. 13, no. 1, pp. 187-197, Feb. 2005.

[5] A. Giorgetti, N. Sambo, L. Valcarenghi, and P. Castoldi, "Reservation collision avoidance in GMPLS wavelength-routed optical networks," in Proc. Photonics in Switching PS '06, Herakleion, Greece, Oct. 2006.

[6] N. Sambo, A. Giorgetti, I. Cerutti, and P. Castoldi, "A contention detection scheme for lightpath restoration in GMPLS networks," IEEE Commun. Lett., vol. 11, no. 10, pp. 820-822, Oct. 2007.

[7] X. Yuan, R. Melhem, and R. Gupta, "Distributed path reservation algorithms for multiplexed all-optical interconnection networks," IEEE Trans. Comput., vol. 48, no. 12, pp. 1355-1363, Dec. 1999.

[8] S. Pitchumani, I. Cerutti, and A. Fumagalli, "Destination-initiated wavelength-weighted reservation protocols: Scalable solutions for WDM rings," in Proc. IEEE Int. Conf. Communications (ICC), Seoul, Korea, May 2005.

[9] S. Arakawa, Y. Kanitani, M. Murata, and K. Kitayama, "A wavelength assignment method for distributed wavelength-routed networks using a circular wavelength-list," in Proc. Broadband Networks, Oct. 2005.

[10] J. Teng and G. N. Rouskas, "Wavelength selection in OBS networks using traffic engineering and priority-based concepts," IEEE J. Select. Areas Commun., vol. 23, no. 8, pp. 1658-1669, Aug. 2006.

[11] S. Ramamurthy and B. Mukherjee, "Survivable WDM mesh networks," J. Lightw. Technol., vol. 21, no. 4, pp. 870-883, Apr. 2003.

[12] N. Andriolli, J. Buron, S. Ruepp, F. Cugini, L. Valcarenghi, and P. Castoldi, "Label preference schemes in GMPLS controlled networks," IEEE Commun. Lett., vol. 10, no. 12, pp. 849-851, Dec. 2006.

[13] R. Braden and L. Zhang, Resource ReSerVation Protocol (RSVP)—Version 1 Message Processing Rules, IETF web site, RFC 2209, Sep. 1997, status: proposed standard.

[14] M. Ghizzi, "Assessment of Selected Features of GMPLS and MTIT Protocols for Traffic Engineering in Core and Metro Segments of the Internet," Ph.D. dissertation, Scuola Superiore Sant'Anna, Pisa, Italy, 2006. 


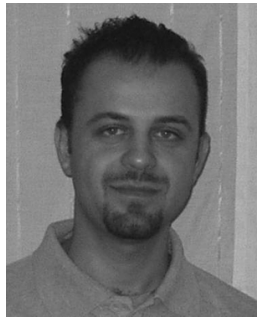

Alessio Giorgetti received the Laurea degree in telecommunications engineering from the University of Pisa, Pisa, Italy, in 2002 and the Ph.D. degree from the Scuola Superiore Sant'Anna, Pisa, in 2006.

In 2007, he was a Visiting Scholar at the Centre for Photonic Systems, University of Cambridge, Cambridge, UK. He is currently a Postdoctoral Fellow with the Scuola Superiore Sant'Anna. His research interests include wavelength-division-multiplexing network design, fault tolerance in optical networks, generalized multiprotocol label switching control plane for wavelength-routed optical networks, next-generation synchronous digital hierarchy technologies, and WDM-passive optical network access networks design.

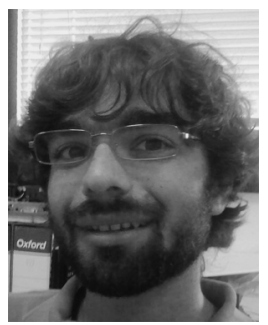

Nicola Sambo (S'06) received the Laurea degree (cum laude) in telecommunication engineering from the University of Pisa, Pisa, Italy in 2004. He is currently pursuing the Ph.D. degree at the Center of Excellence for Information and Communication Engineering (CEIIC), Scuola Superiore Sant'Anna, Pisa.

In 2007, he was a Visiting Student at France Télécom, Lannion. His research interests include WDM network planning and modeling, QoS and fault tolerance in optical networks, and generalized multiprotocol label switching protocol suite for optical networks.

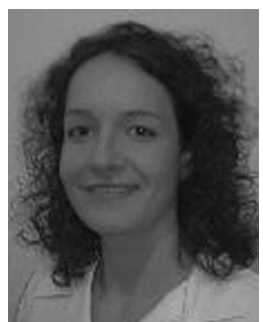

Isabella Cerutti (M'03) received the Laurea degree in electrical engineering from the Politecnico di Torino, Torino, Italy, in 1998 and the Ph.D. degree in electrical engineering from the University of Texas at Dallas in 2002.

From 2002 to 2005, she was a Postdoctorate Research Associate at the University of Texas at Dallas. Currently, she is an Assistant Professor at the Center of Excellence for Information and Communication Engineering (CEIIC), Scuola Superiore Sant'Anna, Pisa, Italy. Her research interests include planning, optimization, performance evaluation, and protocol design of wireless and optical networks.

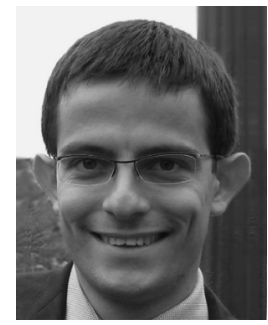

Nicola Andriolli received the Laurea degree in telecommunications engineering from the University of Pisa, Pisa, Italy, in 2002, and the Diploma and $\mathrm{Ph} . \mathrm{D}$. degrees from the Scuola Superiore Sant'Anna, Pisa, in 2003 and 2006, respectively.

He was a Visiting Student at Budapest University of Technology and Economics (BUTE), Budapest, Hungary, and at the Department of Communications, Optics and Materials of the Technical University of Denmark (COM-DTU), Copenhagen, and a Visiting Trainee at the National Institute of Information and Communications Technology (NICT), Tokyo, Japan. He is currently an Assistant Professor with the Scuola Superiore Sant'Anna. His research interests include network modeling and simulation, QoS and fault tolerance in optical networks, generalized multiprotocol label switching architecture, and optical packet switching.

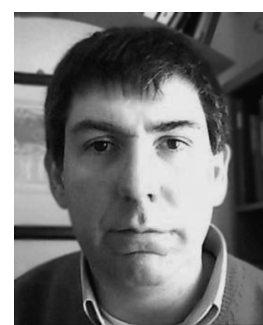

Piero Castoldi (S'93-A'96-M'07) received the Laurea degree (cum laude) in electrical engineering from the University of Bologna, Bologna, Italy, in 1991 and the Ph.D. degree from the University of Parma, Parma, Italy, in 1996.

In 1996 and 1997 and in the summers of 1999 and 2000, he was a Postdoctorate Researcher in the Department of Electrical Engineering, Princeton University, Princeton, NJ. From December 1997 to February 2001, he was an Assistant Professor at the Faculty of Engineering, University of Parma. Since March 2001, he has been an Associate Professor in telecommunications with the Scuola Superiore Sant'Anna, Pisa, Italy, where he is currently responsible for the networks area. In January and February 2002, he was a Visiting Professor at the University of Texas at Dallas. He also collaborates in several projects of the Inter-University National Consortium for Telecommunications (CNIT), and since March 2003, he has been the Director of the CNIT National Photonic Networks Laboratory, Pisa. As an Instructor, he has held several courses at the undergraduate and graduate level at the University of Parma and at Scuola Superiore Sant'Anna. His scientific activity has covered the area of digital transmission, telecommunication-network performance, and, more recently, provisioning, protection, and switching techniques for the next generation optical networks. He is the author of more than 100 technical papers published in international conferences and journals and of an international book on code division multiple access. 